\section{Caracterización de eventos de hipoglicemia en pacientes diabéticos y no diabéticos atendidos en un servicio de urgencia}

\author{
CECILIA VARGAS R..$^{1,2}$, FERNANDO SAN CRISTÓBAL ${ }^{3, a}$, \\ PAULA JARA ${ }^{3, a}$, SEBASTIÁN LÓPEZ ${ }^{3, a}$, JUAN TRUJILLO ${ }^{3, a}$
}

\section{Hypoglycemia in an emergency room. Review of 251 cases}

Background: Hypoglycemia is the main limitation for the achievement of glycemic goals in the treatment of diabetes. Aim: To assess the incidence of hypoglycemia in an emergency department. To characterize and identify which patients are at higher risk of having it. Material and Methods: We reviewed the electronic records of patients discharged from an adult emergency room with the diagnosis of hypoglycemia between May 2011 and December 2014. Age, sex, diagnosis of diabetes (DM), antidiabetic therapy, glycosylated hemoglobin, creatinine, destination at time of discharge, blood glucose, impairment of conscience, treatment of the event and predictions were recorded. Results: Of 175,244 attentions analyzed, 251 in patients aged $69 \pm 17$ years (54\% women) consulted for hypoglycemia (0.14\%). Eighty one percent had a type 2 diabetes, $6 \%$ a type 1 diabetes and 12\% were non-diabetic. Mean blood glucose was $44.1 \mathrm{mg} / \mathrm{dl}$. In diabetic patients, mean glycosylated hemoglobin was 6.5\%. Ninety seven percent had impairment of conscience and $77 \%$ were admitted to the hospital. Among patients without diabetes, the main comorbidity was the history of a gastric bypass surgery. In type 2 diabetes, glibenclamide used alone or with other medications was involved in $59 \%$ of the events, $87 \%$ of patients were older than 65 years with a mean glycosylated hemoglobin of $6.3 \%$ and $32 \%$ had renal failure. Conclusions: The incidence of hypoglycemia was low. There were a significant number of events in older patients with type 2 diabetes mellitus and renal failure, who were treated with glibenclamide. Most of these patients had a glycosylated hemoglobin below accepted recommendations.

(Rev Med Chile 2017; 145: 1387-1393)

Key words: Diabetes Mellitus; Emergency Service, Hospital; Glyburide; Hypoglycemia; Hypoglycemia Agents.
'Servicio de Endocrinología y

Diabetes. Clínica Dávila. Santiago,

Chile.

${ }^{2}$ Sección de Endocrinología y Diabetes. Hospital Clínico Universidad de Chile. Santiago,

Chile.

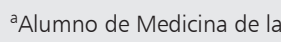
Universidad de los Andes.

Santiago, Chile.

Los autores no declaran conflicto de intereses.

El trabajo no recibió

financiamiento.

Recibido el 20 de abril de 2017, aceptado el 28 noviembre de 2017.

Correspondencia a:

Cecilia Vargas

Macul Alto 6324 casa 19

La Florida.

dra.ceciliavargas@gmail.com
L a literatura médica respalda la importancia de un estricto control glicémico en el paciente con diabetes mellitus (DM) para evitar o disminuir las complicaciones asociadas a la enfermedad. Sin embargo, la mantención de este estricto control glicémico está asociado con un aumento de incidencia de hipoglicemia $(\mathrm{HG})^{1,2}$, lo que representa la limitante principal en el manejo de la $\mathrm{DM}^{3}$.

La HG es un problema frecuente entre usuarios de insulina y antidiabéticos orales que incrementan la concentración de insulina ${ }^{4,5}$. En menor número, puede ocurrir en pacientes sin DM debido a enfermedades endocrinas, renales, hepáticas, 
posterior a ingesta de alcohol y ciertos fármacos ${ }^{5,6}$, siendo reportada también post cirugía bariátrica ${ }^{7}$.

La HG severa se define como aquel episodio de $\mathrm{HG}$ que requiere la intervención de un tercero para su recuperación. La incidencia de HG que requiere asistencia en servicios de emergencia es variable en diversos estudios, pero es mayor en pacientes con diabetes mellitus tipo 1 (DM1) que en diabetes mellitus tipo 2 (DM2) $(2,4$ y 0,4 episodios/100 pacientes/año respectivamente $)^{8}$.

El objetivo de este estudio es conocer la incidencia de HG severa como motivo de consulta de un servicio de urgencia y caracterizar a los pacientes para identificar al grupo en mayor riesgo de desarrollarla.

\section{Material y Método}

Estudio observacional, retrospectivo. Previa aprobación por el comité de ética local, se realiza una revisión sistemática de las fichas clínicas electrónicas de todos los pacientes que egresaron del servicio de urgencia de adultos con el diagnóstico de HG entre mayo de 2011 y diciembre de 2014 inclusive. Nuestro centro es una institución privada de salud, ubicada en la comuna de Recoleta, Región Metropolitana de Santiago, Chile, que atiende a pacientes del sistema privado de salud (Isapre) y del sistema de salud público (Fonasa). El Servicio de Urgencia de Adultos atiende toda la urgencia médico-quirúrgica en mayores de 15 años, excluyendo a aquellas relacionadas con el embarazo y con patología traumatológica, que tienen unidades de atención diferenciadas.

De las fichas electrónicas se obtuvo información de: edad, sexo, tipo de DM, comorbilidades, terapia antidiabética utilizada, hemoglobina glicosilada (HbA1c), glicemia capilar al ingreso, creatinina, velocidad de filtración glomerular (VFG) (según fórmula MDRD-4), definiendo enfermedad renal moderada a severa a aquella con VFG $<60 \mathrm{ml} / \mathrm{min}$. Se consignó además la presencia o no de compromiso de conciencia, tratamiento administrado por la HG, destino al alta (hospitalización o domicilio) y previsión de salud.

Definimos como HG a la presencia de glucosa en sangre menor a $70 \mathrm{mg} / \mathrm{dl}$. HG severa es la que necesita ayuda de un tercero para su recuperación. Compromiso de conciencia es cualquier episodio mencionado como tal o la presencia de confusión, sopor o coma.

Las variables continuas son informadas como promedios con su desviación estándar. Las variables discretas son informadas en número y porcentaje. Las diferencias entre las características basales entre pacientes son analizadas con la prueba t test, estimando como significativa una $\mathrm{p}<0,05$.

\section{Resultados}

Entre mayo de 2011 y diciembre de 2014 se efectuaron 175.244 atenciones en el Servicio de Urgencia de Adultos de Clínica Dávila. Hubo 251 consultas por HG, en 236 pacientes $(0,14 \%)$. Doce pacientes consultaron por más de un evento de HG en el período estudiado.

La mayoría de los pacientes fueron mujeres (54\%) y la edad promedio fue de 68,8 $\pm 17,3$ años (19 a 97 años). El 81\% (205) de los eventos ocurrieron en pacientes con DM2, 12\% (31) en pacientes sin DM y 6\% (15) en DM1. La glicemia capilar era de $44,1 \pm 13,4 \mathrm{mg} / \mathrm{dl}$ y la HbAlc, 6,48 $\pm 1,4 \%$. La creatinina promedio era de $1,41 \pm$ $1,63 \mathrm{mg} / \mathrm{dl}$ y la velocidad de filtración glomerular (VFG, MDRD-4) 77,3 $\pm 44,9 \mathrm{ml} / \mathrm{min}$. El 61\% usaba algún tipo de antidiabético oral (ADO), como mono droga o combinada con insulina u otro ADO. El 28\% usaba insulina como monoterapia. Respecto a la previsión de salud, 62\% eran beneficiarios de Fonasa. En el período de estudio, de los consultantes totales en el servicio de urgencia, $32 \%$ tenía previsión Fonasa $(\mathrm{p}<0,0001)$.

En 97\% (242) de los casos se consignó que el compromiso de conciencia estaba presente al momento de ingresar al servicio de urgencia. Todos los eventos de HG fueron tratados con la administración intravenosa (iv) de glucosa al 30\% (no está especificada cantidad empleada). Se indicó hospitalización en 77,3\% de los casos, pero sólo $62,5 \%$ de los eventos fue finalmente hospitalizado.

Diez pacientes presentaron 2 eventos cada uno, un paciente tuvo 3 y otro 4 eventos. No hubo diferencias significativas entre este grupo de pacientes y aquellos que solo consultaron una vez, en ninguna de las variables analizadas.

En la Tabla 1 se muestran las características de los eventos de HG en pacientes con DM1, DM2 y sin DM. Los DM1 eran menores que los DM2 $(31,3 \pm 11$ vs $73,8 \pm 10,6$ años, $p=0,0001)$. Entre los diabéticos, los DM2 tenían menor HbA1c 
Hipoglicemia en un servicio de urgencia - C. Vargas et al

Tabla 1. Hipoglicemias en pacientes DM1, DM2 y sin DM. Características demográficas, metabólicas, tratamiento de la DM y de la hipoglicemia, destino post recuperación y previsión de salud

\begin{tabular}{|c|c|c|c|c|c|c|c|}
\hline Variable & & $\begin{array}{c}\text { DM1 } \\
(n=15)\end{array}$ & $\begin{array}{c}\text { DM2 } \\
(n=205)\end{array}$ & $\begin{array}{l}\text { No DM } \\
(n=31)\end{array}$ & $\begin{array}{c}\text { DM1 vs } \\
\text { DM2 } \\
p\end{array}$ & $\begin{array}{c}\text { DM1 vs } \\
\text { No DM } \\
p\end{array}$ & $\begin{array}{c}\text { DM2 vs } \\
\text { No DM } \\
p\end{array}$ \\
\hline Edad & (años) & $31,2 \pm 11,7$ & $73,8 \pm 10,6$ & $55,0 \pm 23,3$ & 0,0001 & 0,0010 & 0,0001 \\
\hline Sexo femenino & n (\%) & $9(60,0)$ & $101(49,3)$ & $22(70,9)$ & NS & NS & 0,0491 \\
\hline Glicemia & (mg/dl) & $44,9 \pm 18,0$ & $45,4 \pm 16,5$ & $48,3 \pm 25,1$ & NS & NS & NS \\
\hline $\mathrm{HbA1c}$ & $(\%)$ & $8,28 \pm 3,16$ & $6,36 \pm 0,98$ & $5,55 \pm 0,32$ & 0,0001 & 0,0001 & 0,0001 \\
\hline Creatinina & $(\mathrm{mg} / \mathrm{dl})$ & $1,96 \pm 2,79$ & $1,48 \pm 1,74$ & $1,10 \pm 0,89$ & NS & NS & NS \\
\hline MDRD-4 & $(\mathrm{ml} / \mathrm{min})$ & $107,7 \pm 73,1$ & $72,8 \pm 37,9$ & $105,8 \pm 73,9$ & 0,006 & NS & 0,0001 \\
\hline MDRD-4 $<60(\mathrm{ml} / \mathrm{min})$ & n (\%) & $2(13,3)$ & $65(31,7)$ & $7(22,6)$ & NS & NS & NS \\
\hline \multicolumn{8}{|c|}{ Fármacos antidiabéticos } \\
\hline - Insulina & n (\%) & $14(93,3)$ & $57(27,8)$ & $1(3,2)$ & 0,0001 & 0,0001 & 0,0015 \\
\hline - Insulina + ADO & n (\%) & $1(6,7)$ & $22(10,7)$ & $0(0,0)$ & NS & NS & NS \\
\hline - ADO & n (\%) & $0(0,0)$ & $42(20,5)$ & $6(19,3)$ & NS & NS & NS \\
\hline - 2 ADO o más & n (\%) & $0(0,0)$ & $81(39,5)$ & $0(0,0)$ & 0,001 & NS & 0,0001 \\
\hline - Desconocido & $\mathrm{n}(\%)$ & $0(0,0)$ & $3(1,5)$ & $0(0,0)$ & NS & NS & NS \\
\hline Tratamiento iv de HG & n (\%) & $8(53,3)$ & $145(70,7)$ & $16(51,6)$ & NS & NS & 0,039 \\
\hline \multicolumn{8}{|c|}{ Destino post recuperación de HG } \\
\hline - Hospitalización & n (\%) & $9(60,0)$ & $163(79,5)$ & $21(67,7)$ & NS & 0,0015 & NS \\
\hline - Traslado a otro centro & n (\%) & $1(6,7)$ & $21(12,9)$ & $1(4,8)$ & NS & NS & NS \\
\hline - Cuidados Intensivos & n (\%) & $0(0,0)$ & $2(1,2)$ & $1(4,8)$ & NS & NS & NS \\
\hline - Cuidados Intermedio & $\mathrm{n}(\%)$ & $1(6,7)$ & $87(53,4)$ & $10(47,6)$ & 0,005 & NS & NS \\
\hline - U. Médico Quirúrgica & n (\%) & $6(40,0)$ & $18(11,0)$ & $8(38,0)$ & 0,003 & NS & 0,01 \\
\hline - Rechaza hospitalización & $n(\%)$ & $1(6,7)$ & $35(21,5)$ & $1(4,8)$ & NS & NS & NS \\
\hline - Alta a su domicilio & n (\%) & $6(40,0)$ & $42(20,5)$ & $10(32,3)$ & NS & NS & NS \\
\hline \multicolumn{8}{|l|}{ Previsión de salud } \\
\hline - Fonasa & n (\%) & $7(46,7)$ & $133(64,9)$ & $15(48,4)$ & NS & NS & NS \\
\hline - Isapre & n (\%) & $8(53,3)$ & $69(33,6)$ & $8(25,8)$ & NS & NS & NS \\
\hline - Otro & n (\%) & $0(0,0)$ & $3(1,5)$ & $8(25,8)$ & NS & 0,039 & 0,0001 \\
\hline
\end{tabular}

$(6,36 \pm 0,98 \%$ vs $8,28 \pm 3,16 \%, p=0,0001) y$ menor VFG $(72,8 \pm 44,6$ vs $107,7 \pm 73,1 \mathrm{ml} / \mathrm{min}$, $\mathrm{p}=0,006)$. Hubo diferencias esperadas en el uso de fármacos entre los grupos. No hubo diferencias significativas en la indicación de hospitalización tras la recuperación del evento HG, pero los DM2 se hospitalizaban más en una unidad de pacientes críticos (unidad de tratamiento intermedio) y los DM1 ingresaban más en unidades de cuidados básicos (unidad médico-quirúrgico).

En la Tabla 2 podemos ver las características de los eventos de HG en DM2 según grupo etario. Los mayores de 65 años representaron el 78,5\% (161 pacientes) del total de DM2, los mayores de
75 años al 45,3\% (93 pacientes) y los mayores de 85 años al 7,8\% (16 pacientes). Los pacientes mayores de 65 años fueron mayoritariamente hospitalizados en unidades críticas y no hubo diferencias en la terapia antidiabética entre los grupos.

En los pacientes con DM2 se observó una elevada concentración en torno a la cifra de $\mathrm{HbAlc} \mathrm{de}$ $6,5 \mathrm{mg} / \mathrm{dl}$. El 82,9\% (170 pacientes) tenía HbAlc $\leq 6,5 \mathrm{mg} / \mathrm{dl}$. El $70 \%$ de los pacientes con edad $\leq 65$ años y el 85,6\% de los mayores de 65 años tenía $\mathrm{HbAlc} \leq 6,5 \mathrm{mg} / \mathrm{dl}, \mathrm{p}=0,059$. El 88,9\% de los mayores de 75 años y $88,2 \%$ de los mayores de 85 años tenía $\mathrm{HbAlc} \leq 6,5 \mathrm{mg} / \mathrm{dl}$, sin diferencia significativa. 
Tabla 2. Eventos de hipoglicemia en DM2 según grupo etario y variables evaluadas

\begin{tabular}{|c|c|c|c|c|c|c|c|}
\hline Variable & & $\begin{array}{c}<65 \text { años } \\
(n=25) \\
(1)\end{array}$ & $\begin{array}{c}65 \text { a } 85 \\
(n=163) \\
(2)\end{array}$ & $\begin{array}{c}>85 \text { años } \\
(n=17) \\
(3)\end{array}$ & $\begin{array}{c}1 \text { vs } 2 \\
p\end{array}$ & $\begin{array}{c}1 \text { vs } 3 \\
p\end{array}$ & $\begin{array}{c}2 \text { vs } 3 \\
p\end{array}$ \\
\hline Sexo femenino & n (\%) & $10(40,0)$ & $80(49,1)$ & $11(64,7)$ & NS & NS & NS \\
\hline Glicemia & $(\mathrm{mg} / \mathrm{dl})$ & $46,3 \pm 12,0$ & $44,2 \pm 12,8$ & $39,5 \pm 6,7$ & NS & 0,04 & NS \\
\hline $\mathrm{HbA} 1 \mathrm{c}$ & (\%) & $6,67 \pm 1,34$ & $6,33 \pm 0,93$ & $6,21 \pm 0,94$ & NS & NS & NS \\
\hline MDRD-4 & $(\mathrm{ml} / \mathrm{min})$ & $105,0 \pm 55,5$ & $69,4 \pm 34,9$ & $66,0 \pm 21,3$ & 0,0001 & 0,009 & NS \\
\hline MDRD-4 < $60 \mathrm{ml} / \mathrm{min}$ & n (\%) & $3(12,0)$ & $56(34,3)$ & $6(35,3)$ & 0,035 & NS & NS \\
\hline $\begin{array}{l}\text { Fármacos antidiabéticos } \\
\text { - Insulina } \\
\text { - Insulina + ADO } \\
\text { - ADO } \\
\text { - } 2 \text { ADO o más } \\
\text { - Desconocido }\end{array}$ & $\begin{array}{l}\text { n (\%) } \\
\text { n (\%) } \\
\text { n (\%) } \\
\text { n (\%) } \\
\text { n (\%) }\end{array}$ & $\begin{array}{r}10(40,0) \\
2(8,0) \\
5(20,0) \\
8(32,0) \\
0(0,0)\end{array}$ & $\begin{array}{r}41(25,1) \\
20(12,3) \\
34(20,8) \\
66(40,5) \\
2(1,2)\end{array}$ & $\begin{array}{r}6(35,3) \\
0(0,0) \\
3(17,6) \\
7(41,2) \\
1(5,9)\end{array}$ & $\begin{array}{l}\text { NS } \\
\text { NS } \\
\text { NS } \\
\text { NS } \\
\text { NS }\end{array}$ & $\begin{array}{l}\text { NS } \\
\text { NS } \\
\text { NS } \\
\text { NS } \\
\text { NS }\end{array}$ & $\begin{array}{l}\text { NS } \\
\text { NS } \\
\text { NS } \\
\text { NS } \\
\text { NS }\end{array}$ \\
\hline Tratamiento iv de HG & n (\%) & $17(68,0)$ & $115(70,6)$ & $13(76,5)$ & NS & NS & NS \\
\hline $\begin{array}{l}\text { Destino post recuperación hi } \\
\text { - Traslado a otro centro } \\
\text { - Unidad de paciente crítico } \\
\text { - Unidad médico quirúrgica } \\
\text { - Rechaza hospitalización } \\
\text { - Alta a domicilio }\end{array}$ & $\begin{array}{l}\text { nipoglicem } \\
\text { n (\%) } \\
\text { n (\%) } \\
\text { n (\%) } \\
\text { n (\%) } \\
\text { n (\%) }\end{array}$ & $\begin{array}{r}3(12,0) \\
5(20,0) \\
2(8,0) \\
3(12,0) \\
12(48,0)\end{array}$ & $\begin{array}{r}17(10,4) \\
74(45,4) \\
15(9,2) \\
31(19,0) \\
26(16,0)\end{array}$ & $\begin{array}{r}1(5,9) \\
10(58,8) \\
2(11,8) \\
1(5,9) \\
3(17,6)\end{array}$ & $\begin{array}{c}\text { NS } \\
0,017 \\
\text { NS } \\
\text { NS } \\
0,0007\end{array}$ & $\begin{array}{r}\text { NS } \\
0,02 \\
\text { NS } \\
\text { NS } \\
\text { NS }\end{array}$ & $\begin{array}{l}\text { NS } \\
\text { NS } \\
\text { NS } \\
\text { NS } \\
\text { NS }\end{array}$ \\
\hline $\begin{array}{l}\text { Previsión } \\
\text { - Fonasa }\end{array}$ & n (\%) & $13(52,0)$ & $107(65,6)$ & $13(76,5)$ & NS & NS & NS \\
\hline
\end{tabular}

En relación a la función renal en los DM2, el promedio de la VFG fue de 72,8 $\pm 37,9 \mathrm{ml} / \mathrm{min}$. Casi un tercio de los pacientes tenían menos de 60 $\mathrm{ml} / \mathrm{min}$. El 12\% de los pacientes menores de 65 años y 34\% de los mayores de 65 años tenía una VFG menor a $60 \mathrm{ml} / \mathrm{min}$.

Respecto a la terapia antidiabética, $60 \%$ de los pacientes con DM2 (123 pacientes) usaba fármacos ADO exclusivamente (en monoterapia o en asociación con otros $\mathrm{ADO}$ ), 28\% (57 pacientes) usaba insulina exclusivamente y 10,7\% (22 pacientes) usaba una asociación de insulina y ADO.

Las sulfonilureas (SU), en monoterapia o asociadas con otro ADO o insulina, estuvieron presentes en $60,9 \%$ de los casos, siendo la glibenclamida la más usada $(97,6 \%)$. La metformina como monoterapia o en asociación con sulfonilureas estaba presente en $40,9 \%$ de los casos. En DM2 hubo 6 casos de HG relacionados con el uso exclusivo de metformina, 4 de los cuales no presentaron compromiso de conciencia y 1 ocurrió en un paciente con VFG de $36 \mathrm{ml} / \mathrm{min}$. La insulina basal o basal bolos como terapia exclusiva estaba presente en $27,8 \%$ de los eventos de HG y combinada con 1 o 2 ADO en $10,7 \%$. No hubo diferencias significativas en el uso de fármacos en diversos grupos etarios. Tampoco hubo diferencias significativas entre $\mathrm{HbAlc}$, glicemia de ingreso y los distintos esquemas de tratamiento. Al analizar función renal y tipos de fármacos utilizados, no encontramos diferencias significativas. El 49\% de los pacientes con enfermedad renal moderada a grave estaba siendo tratado con glibenclamida.

En DM2, la glibenclamida sola o en asociación con insulina u otro ADO estuvo presente en $66 \%$ de las hospitalizaciones, mientras que la insulina sola o combinada fue reportada en $26 \%$. Las hipoglicemias relacionadas con SU sola o combinada con insulina u otro ADO causaron más hospitalizaciones que las asociadas con cualquier otro esquema antidiabético en el que la SU no estuvo presente $(90,2 \%$ vs $61,4 \%, p=0,001)$.

El 12\% de las HG severas fueron presentadas por pacientes no diabéticos. Tenían edades inter- 
Tabla 3. Comorbilidades presentes en pacientes no diabéticos con HG

\begin{tabular}{|c|c|c|}
\hline & $n=31$ & $\%$ \\
\hline Bypass gástrico & 5 & 16,1 \\
\hline Enfermedad renal crónica & 5 & 16,1 \\
\hline Resistencia insulínica & 5 & 16,1 \\
\hline Alcoholismo & 3 & 9,7 \\
\hline Neoplasias & 3 & 9,7 \\
\hline Disminución de ingesta & 3 & 9,7 \\
\hline Hipotiroidismo & 2 & 6,5 \\
\hline Embarazo & 2 & 6,5 \\
\hline Insulinoma & 1 & 3,2 \\
\hline Demencia & 1 & 3,2 \\
\hline Daño hepático crónico & 1 & 3,2 \\
\hline Uso de levofloxacino & 1 & 3,2 \\
\hline
\end{tabular}

medias entre los DM1 y DM2, y 71\% eran mujeres. Los niveles de glicemia capilar al ingreso no diferían significativamente respecto de los pacientes con DM ni tampoco se hospitalizaron más que estos. Los pacientes sin DM tenían mejor función renal que los DM. El 77\% no usaba fármacos potencialmente hipoglicemiantes. Hubo un caso de uso de insulina con fines suicidas y 6 usuarios de $\mathrm{ADO}$ ( 5 usaban metformina por resistencia a la insulina o hiperinsulinismo y 1 paciente usaba clorpropamida por diabetes insípida). En la Tabla 3 se consignan las principales comorbilidades que pudieran tener relación con el evento de HG.

\section{Discusión}

La incidencia de HG fue menor a la reportada en otros estudios ${ }^{8-10}$. Lo anterior pudiera ser explicado por una falta probable de registros (dada la naturaleza del estudio) y el hecho de que los pacientes con previsión Fonasa cuenten con centros de la red pública cercanos al nuestro para su atención de emergencia. Sin embargo, cabe destacar que mientras un tercio de todos los pacientes atendidos en el servicio de urgencia pertenecían a Fonasa, este porcentaje fue el doble en las consultas por HG.

En nuestro estudio, el principal grupo de diabéticos que consultaron por HG severa eran DM2 (responsables de $81 \%$ de consultas). Estos pacientes pertenecían a un grupo más añoso, con nivel promedio de $\mathrm{HbA} 1 \mathrm{c}$ de $6,4 \mathrm{mg} / \mathrm{dl}$ y glicemia capilar al momento de la primera atención de 45,5 mg/dl. El 59,5\% usaba algún tipo de ADO, $28 \%$ insulina y $11 \%$ insulina asociada a ADO. Esto difiere de otras series, en que el mayor porcentaje de pacientes DM2 que consultan por $\mathrm{HG}$ son usuarios de insulina ${ }^{4}$. Una explicación de esta diferencia pudiera ser que los pacientes con insulinoterapia reciben más instrucción en cómo tratar las HG ambulatoriamente. Por otro lado, cabe destacar que en Chile y gran parte del mundo, aproximadamente un tercio de los pacientes DM2 es tratado con $\mathrm{SU}^{11,12}$. Sin embargo, a diferencia de lo que ocurre en países desarrollados, en Chile casi la totalidad de este tipo de fármacos es glibenclamida, que tiene un riesgo de hipoglicemia de hasta $83 \%$ más que las otras $\mathrm{SU}^{12-15}$.

Otro aspecto interesante fue encontrar a la metformina como fármaco único asociado a $\mathrm{HG}$, destacando que la mayoría de los casos sin compromiso de conciencia se encontraba en este grupo y que algunos de los eventos podrían atribuirse a otros factores causales.

En nuestra serie se observa que los pacientes mayores de 65 años presentaban mayoritariamente una $\mathrm{HbA1c}$ inferior a $7 \%$ y que aun era más baja en sujetos con mayor edad. Desconocemos la prevalencia de anemia ferropénica que pudiera estar alterando los resultados de la HbAlc, pero se sabe que afecta principalmente a niños y a mujeres en edad fértil ${ }^{16}$, población minoritaria en nuestro estudio. Respecto a la diálisis, presente en 7 casos y que pudiera alterar la confiabilidad en la $\mathrm{HbAlc}$, hay estudios que demuestran que la $\mathrm{HbAlc}$ sigue siendo una medida de control adecuada ${ }^{15}$. Existe evidencia que demuestra que niveles tan estrictos de control metabólico están relacionados con HG y no son aconsejables en pacientes con alto riesgo de presentarlas como los adultos mayores ${ }^{18,19}$. Casi $80 \%$ de todos los ingresos por $\mathrm{HG}$ severa fueron en pacientes mayores de 65 años. En estos pacientes hubo un elevado empleo de glibenclamida como monoterapia o asociada, a pesar de la evidencia que desaconseja su uso en este grupo etario. Otro aspecto destacable es que los pacientes mayores de 65 años tienen peor función renal, lo que se asocia a mayor riesgo para HG. No se aprecia una reducción en el uso de fármacos antidiabéticos 
asociados a mayor riesgo de $\mathrm{HG}$ en pacientes mayores con disfunción renal. La gran mayoría de estos pacientes requirió hospitalización y asistencia en unidades críticas, lo que ha demostrado un significativo impacto financiero en los sistemas de salud ${ }^{9,10,20}$. En nuestro estudio hubo un importante número de pacientes pertenecientes a Fonasa, lo que puede significar un elevado costo en nuestra salud pública.

Respecto a los casos de hipoglicemia en pacientes $\sin \mathrm{DM}$ hay un mayor número que lo descrito en otros estudios (6 y $3 \%$ respectivamente) ${ }^{21,22}$, destacando el antecedente de bypass gástrico y estados patológicos que pudieran estar relacionados con baja ingesta o desnutrición.

\section{Limitaciones}

Es un estudio en un único centro. $\mathrm{Al}$ ser un estudio retrospectivo, y de revisión de fichas electrónicas, no se conoce la causa subyacente de la hipoglicemia. Tampoco se conoce la información de eventos de hipoglicemia (sintomáticos o no) previos a la consulta en el servicio de urgencia. Respecto al tratamiento, solo se consigna el uso de ampollas de solución glucosada a 30\%, pero no la cantidad empleada. No se precisó el grado de compromiso de conciencia presente al ingreso. No se investigó la hospitalización asociada al evento de HG.

\section{Fortalezas}

Este estudio da información de la frecuencia de hipoglicemia grave que ingresa a un servicio de urgencia de una institución privada de salud. Sin embargo, la principal composición de pacientes consultantes son beneficiarios del sistema público de salud de Chile (Fonasa), por lo que no habría un sesgo de la previsión de salud. Aporta, además, información acerca del control metabólico de los pacientes ingresados por $\mathrm{HG}$, destacando que la mayoría tenía un control más estricto de lo que recomiendan las guías y consensos ${ }^{23}$.

\section{Conclusiones}

Las consultas por HG severa en un servicio de urgencia son mayoritariamente de pacientes con DM2, adultos mayores, con un control metabólico por debajo de las recomendaciones y con función renal reducida. Son usuarios de sulfonilureas solas o asociadas, las que no son recomendadas en esta población por su alto riesgo de hipoglicemias. La mayoría de estas consultas termina en una hospitalización en una unidad de paciente crítico, lo que implica un significativo costo para el sistema de salud. Estos hallazgos obligan al clínico a ajustar el tratamiento y las metas terapéuticas en los grupos de pacientes con riesgo de hipoglicemias, especialmente en las personas de la tercera edad, que es un grupo creciente en nuestra población. Se debe enfatizar en el logro de metas glicémicas ajustadas al paciente y terapias antidiabéticas eficientes y seguras, lo que nos obliga a mantener una atención personalizada en todos los pacientes, apoyándonos en las guías clínicas y en la evidencia científica. Otro aspecto destacable es que, dentro del escaso número de pacientes no diabéticos que presentaron una hipoglicemia, surge la cirugía bariátrica como comorbilidad potencialmente causal, además de aquellas condiciones o enfermedades asociadas a la desnutrición. Este hallazgo obliga a poner atención en esta problemática dado el aumento de este tipo de intervenciones quirúrgicas.

\section{Referencias}

1. The Diabetes Control and Complications Trial Research Group. The effect of intensive treatment of diabetes on the development and progression of long-term complications in insulin-dependent diabetes mellitus. NEJM 1993; 329: 977-86.

2. UK Prospective Diabetes Study (UKPDS). Intensive blood-glucose control with sulphonylureas or insulin compared with conventional treatment and risk of complications in patients with type 2 diabetes (UKPDS 33). Lancet 1998; 352: 837-53.

3. Cryer PE, Davis SN, Shamoon H. Hypoglycemia in diabetes. Diabetes Care 2003; 26: 1902-12.

4. Leese G, Wang J, Broomhall J, Kelly P, Marsden A, Morrison W, et al. DARTS/MEMO Collaboration. Frequency of severe hypoglycemia requiring emergency treatment in type 1 and type 2 diabetes: a population-based study of health service resource use. Diabetes Care 2003; 26: 1176-80.

5. Cryer P, Axelrod L, Grossman A, Heller S, Montori V, Seaquist E, et al. Endocrine Society. Evaluation and management of adult hypoglycemic disorders: an Endocrine Society Clinical Practice Guideline. J Clin Endocrinol Metab 2009; 94 (3): 709-28. 
6. Murad M, Coto-Yglesias F, Wang A, Sheidaee N, Mullan $\mathrm{R}$, Elamin M, et al. Clinical review: drug-induced hypoglycemia: a systematic review. J Clin Endocrinol Metab 2009; 94 (3): 741-5.

7. Service G, Thompson G, Service F, Andrews J, CollazoClavell M, Lloyd R. Hyperinsulinemic hypoglycemia with nesidioblastosis after gastric-bypass surgery. N Engl J Med 2005; 353 (3): 249-54.

8. Krnacova V, Kubena A, Macek K, Bezdek M, Smahelova A, Vlcek J. Severe hypoglycaemia requiring the assistance of emergency medical servicies-frecuency, causes and symptoms. Biomed Pap Med Fac Univ Palacky Olomouc Czech Repub 2012; 156 (3): 271-7.

9. Barranco R, Gómez-Peralta F, Abreu C, Delgado M, Palomares R, Romero F, et al. Incidence and care-related costs of severe hypoglycaemia requiring emergency treatment in Andalusia (Spain): the PAUEPAD Project. Diabetic Medicine 2015; 32: 1520-26.

10. Parsaik A, Carter R, Pattan V, Myers L, Hamit K, Smith S, et al. Population-based study of severe hypoglycemia requiring emergency medical service assistance reveals unique findings. J Diabetes Sci Technol 2012; 1: 65-72.

11. Chile. Ministerio de Salud. Evaluación de las características y cambios en el tratamiento de la diabetes en la población general chilena. ENS 2003 y ENS 2009-10. Santiago: MINSAL, disponible en: http://web.minsal.cl/ sites/default/files/files/Informecoberturaefectivadiabetes. pdf [consultado el 23 de diciembre de 2016].

12. Castro R, Estrada L, Goez L. Evaluación de la prescripción de glibenclamida en diabéticos tipo 2. Rev SALUD UIS [Internet] 2017; 49 (491): 9-15. Available from: http://dx.doi.org/10.18273/revsal.v49n1-2017001.

13. Gangji A, Cukierman T, Gerstein H, Goldsmith C, Clase C. A systematic review and meta-analysis of hypoglycemia and cardiovascular events: a comparison of glyburide with other secretagogues and with insulin. Diabetes Care 2007; 30 (2): 389-94.

14. Simpson S, Lee J, Choi S, Abdelmoneim A, Feathetsto- ne T. Mortality risk among sulfonylureas: a systematic review and network meta-analysis. The lancet. Diabetes \& Endocrinology 2015; 3 (1): 43-51.

15. Palmer S, Mavridis D, Nicolucci A, Johnson D, Tonell $\mathrm{M}$, Craig J, et al. Comparison of Clinical Outcomes and Adverse Events Associated With Glucose-Lowering Drugs in Patients With Type 2 Diabetes: A Meta-analysis. JAMA 2016; 316 (3): 313-24.

16. World Health Organization, Center for Disease Control and Prevention Atlanta. Worldwide prevalence of anaemia 1993-2005, WHO Global Database on Anaemia. Geneva, World Health Organization, 2008.

17. López G, Sanzana MG, Durruty P, Sanhueza M, Flores F, Alvo M, et al. Glycosylated hemoglobin and fructosamine as markers of metabolic control among diabetic patients on hemodialysis. Rev Chil Endocrinol Diabetes 2013; 6 (2): 50-4.

18. Kirkman M, Briscoe V, Clark N, Florez H, Haas L, Halter J, et al. Diabetes in older adults. Diabetes Care 2012; 35: 2650-64.

19. Pogach L, Tseng C, Maney M, Aron D. A Proposal for an out-of-range glycemic population health safety measure for older adults with diabetes. Diabetes Care 2017; 40: 518-25.

20. Veronese G, Marchesini G, Forlari G, Saragoni S, Degli Esposti L, Centis E, et al. Costs associated with emergency care and hospitalization for severe hypoglycemia. Nutrition, Metabolism and Cardiovascular Diseases 2016; 26 (4): 345-51.

21. Holstein A, Plaschke A, Vogel M, Egberts E. Prehospital management of diabetic emergencies-a population-based intervention study. Acta Anaesthesiol Scand 2003; 47: 610-5.

22. Socransky S, Pirrallo R, Rubin J. Out-of-hospital treatment of hypoglycemia: refusal of transport and patient outcome. Acad Emerg Med 1998; 5: 1080-5.

23. American Diabetes Association. Standards of Medical Care in Diabetes. Diabetes Care 2017; 40 (Suppl. 1): S48-S56. 